\title{
Individual differences in discount rate are associated with demand for self-administered cocaine, but not sucrose
}

\author{
Mikhail N. Koffarnus' \& James H. Woods² \\ Johns Hopkins University School of Medicine, Department of Psychiatry, Baltimore, MD, USA' and University of Michigan, Department of Pharmacology, \\ Ann Arbor, MI, USA²
}

\begin{abstract}
Substance abusers, including cocaine abusers, discount delayed rewards to a greater extent than do matched controls. In the current experiment, individual differences in discounting of delayed rewards in rats (choice of one immediate over three delayed sucrose pellets) were assessed for associations with demand for either sucrose pellets or an intravenous dose of $0.1 \mathrm{mg} / \mathrm{kg} / \mathrm{infusion}$ cocaine. Twenty-four male Sprague Dawley rats were split into three groups based on sensitivity to delay to reinforcement. Then, demand for sucrose pellets and cocaine was determined across a range of fixed-ratio values. Delay discounting was then reassessed to determine the stability of this measure over the course of the experiment. Individual differences in impulsive choice were positively associated with elasticity of demand for cocaine, a measure of reinforcer value, indicating that rats having higher discount rates also valued cocaine more. Impulsive choice was not associated with the level of cocaine consumption as price approached 0 or with any parameter associated with demand for sucrose. Individual sensitivity to delay was correlated with the initial assessment when reassessed at the end of the experiment, although impulsive choice increased for this cohort of rats as a whole. These findings suggest that impulsive choice in rats is positively associated with valuation of cocaine, but not sucrose.
\end{abstract}

Keywords Behavioral economics, cocaine demand, delay discounting, impulsive choice, impulsivity, Sprague Dawley rats.

Correspondence to: Mikhail N. Koffarnus, Johns Hopkins University School of Medicine, Department of Psychiatry, 5200 Eastern Ave., Suite W142, Baltimore, MD 21224, USA. E-mail: mkoffar1@jhmi.edu

Impulsivity is a construct that is commonly split into at least two types of impulsive behavior: impulsive choice or inter-temporal choice and what is termed impulsive action or behavioral inhibition (Evenden 1999; Winstanley, Eagle \& Robbins 2006; Dalley et al. 2008; Perry \& Carroll 2008; de Wit 2009). Impulsive choice is the tendency to choose smaller rewards delivered sooner over larger rewards delivered later, while impulsive action refers to the inability to withhold or inhibit a pre-potent response. Impulsive choice is typically measured using procedures that provide choice opportunities between a smaller amount of a reinforcer delivered after little or no delay and larger amounts of the same reinforcer delivered after a longer delay (Ainslie 1975). Animal and human models of impulsive choice are widely studied, and in humans, extensive evidence links delay discounting to substance abuse. Substance abusers demonstrate a higher degree of impulsive choice than do matched controls across a range of substances, including cocaine (Coffey et al. 2003; Kirby \& Petry 2004).

Despite the robust relationship between delay discounting and substance abuse in people, relatively little research has examined the analogous relationship between delay discounting and drug self-administration in animals. Animal-subjects research offers many opportunities not available in human-subjects research, including the ability to easily measure behavioral characteristics both before and after a subject is exposed to a drug like cocaine. Delay discounting is modeled straightforwardly in animals with procedures that offer choices between varying amounts of some reinforcer available at varying delays (Mazur 1987; Evenden \& Ryan 1996; 
Richards et al. 1997). Impulsive choice is increased after a period of self-administered cocaine (Mendez et al. 2010), and non-contingent exposure to cocaine has been shown to produce lasting (Simon, Mendez \& Setlow 2007) or transient (Logue et al. 1992; Paine, Dringenberg \& Olmstead 2003) increases in impulsive choice. Individual differences in impulsive choice also predicted acquisition of cocaine self-administration in female rats (Perry et al. 2005), as well as level of nicotine selfadministration at high response requirements and reinstatement of extinguished nicotine self-administration in male rats (Diergaarde et al. 2008). It is not clear, however, whether animals that discount delayed rewards steeply value these drug reinforcers to a greater extent, as response rate-based measures of drug reinforcement have many shortcomings (for recent reviews, see Bergman \& Paronis 2006; Hursh \& Silberberg 2008). When self-administered, different doses of the same drug support different amounts of behavior, with moderate doses typically supporting more behavior than both high and low doses (Bergman \& Paronis 2006). This feature makes dose an influential variable when assessing reinforcer value, with no clear method of choosing which dose of a given drug best represents the reinforcing value of that drug. In addition, many self-administered drugs function to increase or decrease general activity, confounding the independent variable being assessed (e.g. drug A versus drug B) and the dependent measure (e.g. lever presses maintained by drug A versus drug B).

Behavioral economics, the application of economic terms, concepts and analytical tools to the study of the behavior of individual organisms (Bickel, Green \& Vuchinich 1995), provides a system of assessing reinforcer value that is independent of drug dose (Hursh \& Silberberg 2008). In such an analysis, total consumption of a reinforcer, rather than response rate, is the dependent measure, and this is determined at a variety of prices (response requirements). The rate at which consumption declines as price increases is termed the elasticity of demand, and this measure is assumed to reflect the reinforcer value. When demand of a reward is inelastic, an individual will defend its consumption of that reward even as price is increased dramatically, leading to large increases in total effort expended to consume that reward. As a metric of reinforcer value, elasticity of demand goes beyond a response rate-based measure by more fully characterizing the relationship between the price of a reinforcer and its consumption. Elasticity of demand can be used to rank order reinforcer value across different reinforcers (e.g. Hursh \& Winger 1995) or across different environmental conditions with responding maintained by the same reinforcer (e.g. Hursh 1991). Note that elasticity of demand depends only on rate of decline in consumption and does not depend on total level of responding, total consumption or the dose of the selfadministered drug. Elasticity of demand therefore avoids many of the potential confounds introduced by rate of responding measures when assessing value of selfadministered drugs.

In the current experiment, individual differences in discounting rate, assessed by a procedure developed by Evenden \& Ryan (1996), were related to individual differences in valuation of sucrose pellets and cocaine injections, assessed using behavioral economic demand analysis. The relationship between cocaine use and steep delay discounting is consistently found in human-subjects research (Coffey et al. 2003; Kirby \& Petry 2004), and the demonstration of a similar relationship in experimental animals would provide a framework for determining the nature of this relationship, including whether treatments that reduce impulsive choice could also reduce the valuation of drugs of abuse. Based on the strong positive relationship between drug abuse and impulsive choice in humans, a similar positive relationship was hypothesized between delay discounting rate and demand for cocaine in rats in the present experiment. Delay discounting measures were also assessed for stability over the course of the experimental procedure, with a delay discounting reassessment after demand determination.

\section{METHOD}

\section{Subjects}

Twenty-four male Sprague Dawley rats served as subjects (Harlan, Indianapolis, IN). Rats were approximately 10 weeks old at the start of the experiment and approximately 46 weeks at the end of the experiment. Weights were maintained at approximately $325 \mathrm{~g}$ throughout all four phases of the experiment, which is approximately $85 \%$ of the mean adult weight supplied by the manufacturer for this strain. When not in session, rats were individually housed in accordance with institutional animal care and use guidelines in polycarbonate cages with fresh water continuously available. The lights in the housing colony were on from 7:00 AM to 7:00 PM, and sessions were conducted between 9:00 AM and 5:00 PM. These protocols were approved by the University of Michigan Committee on the Use and Care of Animals and conformed to the guidelines established by the National Institutes of Health (NIH) Guide for the Use of Laboratory Animals.

\section{Apparatus}

Sessions were conducted in rodent operant conditioning chambers (ENV-008) which, along with all other components, were supplied by Med Associates Inc. (St. Albans, VT, USA). Both sides of the front panel of the chamber held a retractable lever (ENV-112CM). Between the levers 


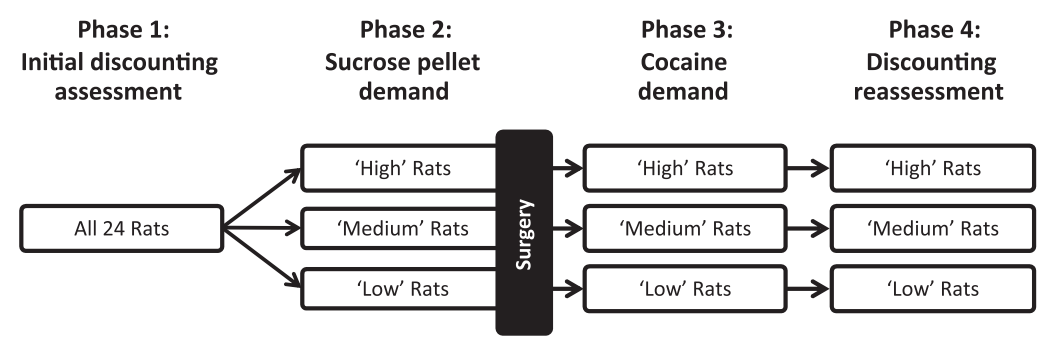

Figure I A schematic of the study design. All rats experienced four phases in the order shown in the figure. Rats were split into groups after the phase I discounting assessment, but all rats were treated equally and exposed to the same conditions throughout the entire experiment. See the Procedure section for a detailed description of this progression through the experiment

was a food tray connected to a 45-mg pellet dispenser (ENV-200R1AM and ENV-203 M-45). Above both the levers and the food tray were triple stimulus lights containing a red, green and yellow light-emitting diode (LED; ENV-222 M). Centered on the opposite wall was a nosepoke response device containing a yellow LED (ENV$114 \mathrm{BM}$ ) and a houselight near the top of the wall (ENV$215 \mathrm{M}$ ), which was unused in the current procedure. A syringe pump was located outside the chamber for drug deliveries (PHM-107). Medical-PC IV software (Med Associates) was used to control experimental events and record data.

\section{Procedure}

See Fig. 1 for a schematic of the entire experimental procedure. Rats were initially trained to respond on a conjoint fixed-time 60 seconds fixed-ratio (FR) 1 schedule of reinforcement, with the active lever alternating each session. This schedule arranged one sucrose pellet to be delivered every 60 seconds independent of behavior, with every lever press also producing a pellet. After four sessions, no more response-independent pellets were delivered. The FR 1 schedule continued until all subjects emitted 80 responses or more on two consecutive 20-minute sessions, which took 12 sessions.

\section{Phase 1: initial delay discounting assessment}

The sessions were then extended to 75 minutes and split into five components of 10 discrete-choice trials each. Total trial duration was 90 seconds and began with one or both levers extending into the chamber. If a single response was made within 20 seconds, the levers retracted and the consequence programmed for that lever was delivered. If no response was made within 20 seconds, that trial was recorded as an omission and the levers retracted for the remaining 70 seconds of that trial. The first two trials of each component were always forced-choice trials where only one lever was extended into the chamber, forcing the subject to sample the contingencies for that component. The remaining eight trials were free-choice trials where both levers were extended into the chamber, allowing the rat to respond on either. The red stimulus light above each lever was lit whenever that lever was inserted in the chamber, with the left light constant and the right light flashing at $2 \mathrm{~Hz}$. The green and yellow stimulus lights above the pellet tray were lit during sucrose pellet deliveries. Initially, the consequences for the left and right levers were immediate deliveries of either one or three 45-mg sucrose pellets, respectively. This condition was continued until all the rats chose the three-pellet option on at least $85 \%$ of free-choice trials (seven sessions in total). At this point, delays were introduced between responses made on the three-pellet lever and the delivery of the three pellets. The delays to the three-pellet option were $0,10,20,40$ or 60 seconds and were always presented in ascending order with one delay in effect in each of the five 10-trial components. Rats were exposed to this procedure for 48 sessions.

\section{Phase 2: sucrose pellet demand}

Demand for 45-mg sucrose pellets was then determined. Levers remained retracted throughout this procedure and the nose-poke on the back wall of the chamber was the active response device. The response was changed to a nose-poke for the demand assessments to differentiate this task from the discounting task and to avoid confounding responses on one of the discounting levers with the demand contingencies. At the start of the 30-minute sessions, the nose-poke device was lit and reinforcers were delivered on an FR schedule. The same FR schedule remained in effect for the entire session, but the FR schedule value changed between sessions. The consequence of each completed FR was a brief flash of the yellow and green stimulus lights above the pellet tray, the nose-poke light extinguishing and a single 45-mg sucrose pellet delivered to the tray. After a 5-second timeout period, the nose-poke was illuminated and the FR schedule was again active. FR values of $1,3,10,32$ and 100 were examined in an ascending order. This sequence was repeated three times, with an extra FR 1 session before the first sequence only, for a total of 16 sessions. 


\section{Catheter surgery}

Each rat was then implanted with an indwelling femoral vein catheter for intravenous (i.v.) infusion of cocaine. Rats were surgically prepared under ketamine $[100 \mathrm{mg} /$ $\mathrm{kg}$, intramuscular (i.m.)] and xylazine $(5 \mathrm{mg} / \mathrm{kg}$, i.m.) anesthesia. The surgical field was shaved and cleaned with povidone iodine, and ocular lubricant was applied to the eyes prior to the beginning of the surgery. A small incision was made just above the femoral vein, and the overlying tissue was dissected. The wound was closed using 5-0 Ethilon suture (Ethicon, San Angelo, TX, USA), and the catheters were tunneled under the skin and attached to a stainless steel tubing, exiting the back through a Dacron mesh tether button (DC95B, Instech Laboratories, Inc., Plymouth Meeting, PA, USA) that was sutured to the muscle between the scapula. Rats were allowed 5-7 days to recover from surgery prior to the resumption of the experiment. Catheters were flushed daily with $0.25 \mathrm{ml}$ of saline containing heparin (100 U/ $\mathrm{ml})$ to promote catheter patency.

\section{Phase 3: cocaine demand}

Rats were initially allowed to respond for contingent infusions of $0.56 \mathrm{mg} / \mathrm{kg} /$ infusion cocaine (National Institute on Drug Abuse, Bethesda, MD, USA) on the nose-poke device on an FR 1 schedule. This continued until rats self-administered at least 20 infusions of cocaine in a 60-minute session (one subject never met this criterion). The dose of cocaine was then lowered to $0.1 \mathrm{mg} / \mathrm{kg} /$ infusion, and rats were allowed to self-administer this dose for two sessions on an FR 1 schedule. The session length was then shortened to 30 minutes and cocaine demand was assessed as it was with sucrose pellets. The FR sequence for cocaine demand was 1, 3, 10, 18 and 32, and this sequence was repeated three times for most rats. Fewer repetitions were conducted for some rats that experienced catheter patency problems before three repetitions were complete $(n=10)$. Of those, three completed no full FR sequences and therefore have no cocaine selfadministration data presented. The mean number of total self-administration sessions was 28.15 [standard deviation $(\mathrm{SD})=3.59]$.

\section{Phase 4: delay discounting redetermination}

Rats were then allowed to respond on the delay discounting procedure as described above for 43 sessions.

\section{Data analysis}

Choice data from each rat, expressed as percent choice of three pellets at each delay to three pellets out of the total choices made (omissions were excluded), were analyzed in Prism 5 (GraphPad Software Inc., La Jolla, CA, USA).
To be included in the data for group analysis, delay had to significantly affect choices for the individual subject. This criterion included a significant main effect of delay on choices, determined by a one-way repeated measures analysis of variance (ANOVA) over the last five delay discounting sessions. These ANOVAs assessing the effect of delay on choices were repeated for each subject and were not inferential in that they were not intended to indicate anything beyond the behavior patterns of individual subjects. In addition, three-pellet choice in the 60-second delay condition had to be significantly lower than in the 0 -second delay condition, as measured by a planned post hoc comparison test. These criteria were in place due to a similar rationale to that presented in Johnson \& Bickel (2008). A basic assumption of this research is that reinforcer delay is inversely related to reinforcer value, with the relevant characteristic being the degree of this inverse relationship. For rats that did not show a consistent effect of delay or showed an increased choice of delayed rewards, there is a distinct likelihood that they were not under discriminative control of the relevant contingency (delay to reinforcement). The ANOVAs with the planned post hoc comparisons were used to measure whether such a pattern existed for each subject. For purposes of group formation, choice data were then fit to the hyperbolic equation developed by Mazur (1987) to analyze delay discounting data:

$$
V=\frac{A}{1+k D}
$$

where $V$ is the percent choice of three pellets at $D$ delay, and $A$ and $k$ are fit parameters. $A$ is the derived percent choice of three pellets when delayed 0 seconds, and $k$ is a measure of the effect of delay on choices. $A$ was fitted instead of set to a fixed value because, in practice, it was often less than the predicted proportion of 1 , and it also provided an index of discriminability of the amount of pellets delivered.

Demand functions for sucrose pellets and cocaine infusions were plotted as reinforcers earned as a function of response requirement, known as a demand analysis (Hursh \& Winger 1995; Hursh \& Silberberg 2008). Number of reinforcers was reported as total responses divided by FR value. Data were fit with non-linear regression to the exponential equation

$$
Y=\log L * e^{-a X}
$$

where $Y$ is reinforcer consumption at $X$ price and $L$ and $a$ are fit parameters (Winger et al. 2006). L represents the derived level of unconstrained consumption under the experimental conditions and is typically nearly equal to consumption at an FR 1. The $a$ parameter indicates the elasticity of the curve, or the rate that consumption declines with increases in price. 
To better isolate the elasticity parameter, normalized demand curves were also compared. Consumption data were normalized to a proportion of consumption at an FR 1 with a procedure described previously (Hursh \& Winger 1995). The price that supported the most overall responding was also computed. This value, $P_{\max }$, is directly related to elasticity and can be derived from the a parameter of Equation 2 with the equation $P_{\max }=$ 0.29/a. Pearson correlations were conducted in Prism 5. Distributions of $k$ values were log-transformed to preserve a normal distribution. The best-fit parameters of demand functions were compared between groups using nonlinear regression analyses in Prism 5, which are mathematically equivalent to an analysis of covariance (Motulsky \& Christopoulos 2003).

To address whether response rates tended to increase or decrease within cocaine and sucrose demand sessions, a whole-session index of curvature (Fry, Kelleher \& Cook 1960) was calculated for each session. Each session was split into ten 3-minute components, and the index of curvature quantifies the degree to which response rates tended to increase or decrease across components. This index ranges from +1 (maximum positive acceleration) to -1 (maximum negative acceleration), with 0 indicating a steady response rate. For each subject, index of curvature values were averaged across all FR values and compared across conditions with a paired $t$-test in Prism 5.

\section{RESULTS}

Phase 1: initial discounting assessment

At the end of the phase 1 initial discounting assessment, 20 of the 24 rats met the statistical criteria for inclusion in a discounting group. These 20 rats were ranked by their individual $k$ value from Equation 1 and split into three groups based on the following ranking: high $(n=7)$, medium $(n=6)$ and low $(n=7)$. The choice to use three equally sized groups was made prior to the experiment, as it allowed for more potential differentiation between the high and low groups than a median split. The percent choice of three pellets at each delay to three pellets is shown for the last five sessions of the initial discounting assessment in Fig. 2 (top panel). A two-way ANOVA revealed an overall main effect of delay on choice $\left(F_{4,72}=61.42, P<0.001\right)$ and a main effect of discounting group $\left(F_{2,72}=5.72, P=0.012\right)$. The choices of the three groups were similar when the delay to three pellets was 0 seconds, with a difference among the groups emerging at higher delays. This pattern resulted in a significant delay by discounting group interaction $\left(F_{8,72}=7.35\right.$, $P<0.001)$. Individual discounting functions were generally well described by Equation 1 , with median $r^{2}=0.828$

\section{Initial discounting assessment}
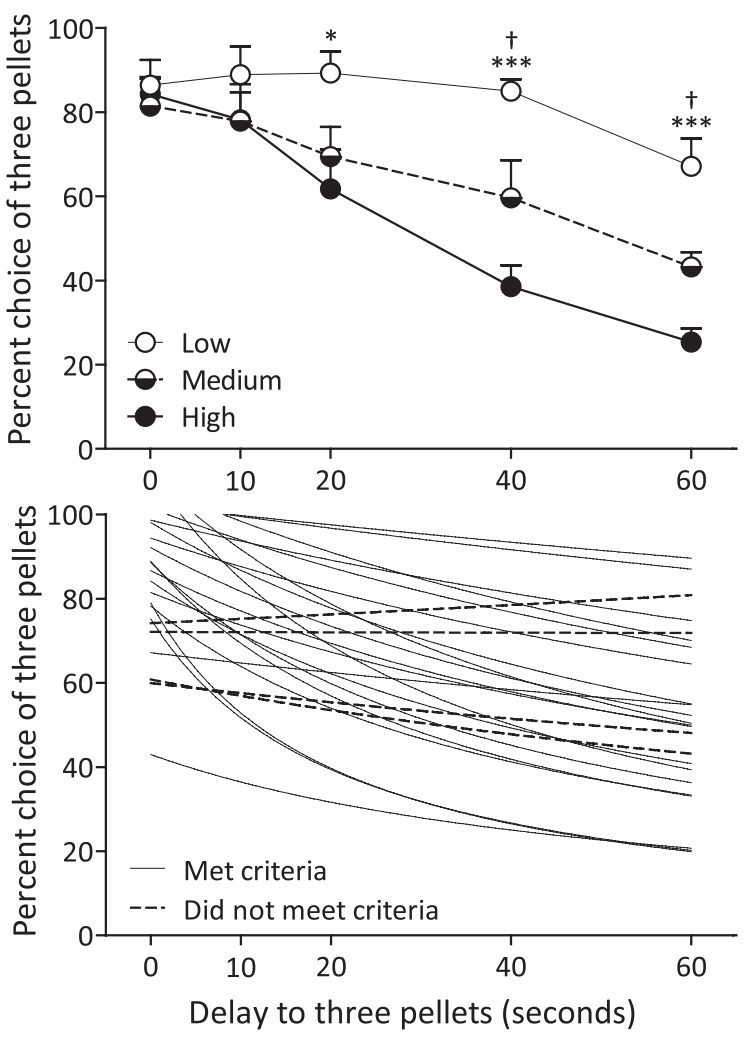

Figure 2 Choice data from the initial delay discounting assessment. Top panel: Groups of rats divided based on the $k$ parameter from Equation I fit to the individual subject data. Data are presented as percent choice of three pellets as a function of the delay to three pellets for the high (filled symbols), medium (half-filled symbols) and low (open symbols) groups. Symbols near points indicate that point is significantly different from the corresponding point in the high ( $* P<0.05$, $* * * P<0.00$ I) or the medium group $(+P<0.05)$, as measured by a Bonferroni-adjusted post hoc test. Bottom panel: The curves obtained by fitting Equation I to the individual subject data. Curves in solid lines represent the 20 rats for which delay significantly affected their choice behavior, as described in the Data analysis section. Curves in broken lines represent the four rats for which delay did not significantly reduce choice behavior. These four rats are not included in the groups in the top panel

(interquartile range $=0.151$ ) for subjects meeting criteria (Fig. 2, bottom panel). Mean $k$ values ( +1 SD and -1 SD in parentheses, which were asymmetrical because means and SD values were based on log-transformed $k$ values) for the high, medium and low groups were $0.030(0.042-$ $0.021)$, $0.014(0.017-0.011)$ and 0.0050 (0.0085$0.0029)$, respectively. Mean $A$ values (SD in parentheses) for the high, medium and low groups were $0.90(0.15)$, $0.81(0.20)$ and $0.96(0.13)$, respectively.

\section{Phase 2: sucrose pellet demand}

Demand for sucrose pellets was then assessed in all 24 rats, with the 20 rats that made up the three 
Table 1 A comparison of the demand parameters for each group of rats split on the basis of the individual $k$ values obtained from the initial discounting assessment. Normalized and standard demand analyses are presented with both sucrose and cocaine reinforcers, as well as the results of $F$ tests comparing these parameters across groups. Statistically significant results appear in bold.

\begin{tabular}{|c|c|c|c|c|c|c|c|}
\hline & \multirow[b]{2}{*}{ Parameter } & \multicolumn{3}{|c|}{ Initial discounting group } & \multirow[b]{2}{*}{ F } & \multirow{2}{*}{$\begin{array}{l}\text { Degree of } \\
\text { freedom }\end{array}$} & \multirow[b]{2}{*}{$\mathrm{P}$} \\
\hline & & Low & Medium & High & & & \\
\hline \multirow[t]{3}{*}{ Sucrose: not normalized } & $L$ & 191 & 198 & 179 & 0.588 & 2,94 & 0.557 \\
\hline & $a$ & 0.00629 & 0.00640 & 0.00644 & 0.0364 & 2,94 & 0.964 \\
\hline & $P_{\max }$ & 46.1 & 45.3 & 45.0 & & & \\
\hline \multirow[t]{2}{*}{ Sucrose: normalized } & $a$ & 0.00391 & 0.00387 & 0.00416 & 0.535 & 2,97 & 0.588 \\
\hline & $P_{\max }$ & 74.2 & 75.0 & 69.6 & & & \\
\hline \multirow[t]{3}{*}{ Cocaine: not normalized } & $L$ & 72.8 & 61.3 & 53.8 & 0.214 & 2,73 & 0.808 \\
\hline & $a$ & 0.0889 & 0.0846 & 0.0455 & 3.53 & 2,73 & 0.034 \\
\hline & $P_{\max }$ & 3.26 & 3.43 & 6.37 & & & \\
\hline \multirow[t]{2}{*}{ Cocaine: normalized } & $a$ & 0.118 & 0.108 & 0.0660 & 5.23 & 2,76 & 0.007 \\
\hline & $P_{\max }$ & 2.47 & 2.68 & 4.39 & & & \\
\hline
\end{tabular}

$a=$ Demand elasticity parameter from Equation 2; $L=$ Parameter representing level of responding from Equation $2 ; P_{\max }=$ Price supporting the maximum level of responding.

discounting groups analyzed in detail. Demand for sucrose pellets was described well by Equation 2 with median $r^{2}=0.966 \quad$ (interquartile range $=0.027$ ). Demand did not differ among the three groups when either the $L$ parameter or the $a$ parameter was compared with curve-fitting procedures (Table 1; Fig. 3, top panel). The corresponding $P_{\max }$ values for each of the three groups were nearly identical (Table 1). Normalized demand curves were also similar among the discounting groups (Table 1; Supporting Information Fig. S1, top panel), and Equation 2 provided a good fit to the data with median $r^{2}=0.968$ (interquartile range $=0.032$ ). The mean index of curvature for sucrose sessions was -0.10 , indicating a slight deceleration of responding within sucrose sessions.

\section{Phase 3: cocaine demand}

Unlike demand for sucrose pellets, demand for i.v. infusions of $0.1 \mathrm{mg} / \mathrm{kg} /$ infusion cocaine did differ as a function of group (Table 1; Fig. 3, bottom panel). The high discounting group had less elastic demand than the low or medium group, which was reflected by a significant difference in the $a$ parameter of Equation 2 fit to these data. The $L$ parameter was not different between groups, however, indicating this group difference was restricted to higher FR values. The corresponding $P_{\max }$ value for the high group (6.37) was considerably higher than the low (3.26) and medium (3.43) groups, and Equation 2 provided a good fit to the cocaine demand data (median $r^{2}=0.961$, interquartile range $\left.=0.077\right)$. When cocaine demand curves were normalized, demand in the high group was still less elastic than in the low and medium groups (Table 1; Supporting Information Fig. S1, bottom panel), and the normalized curves were also well

\section{Demand curves}
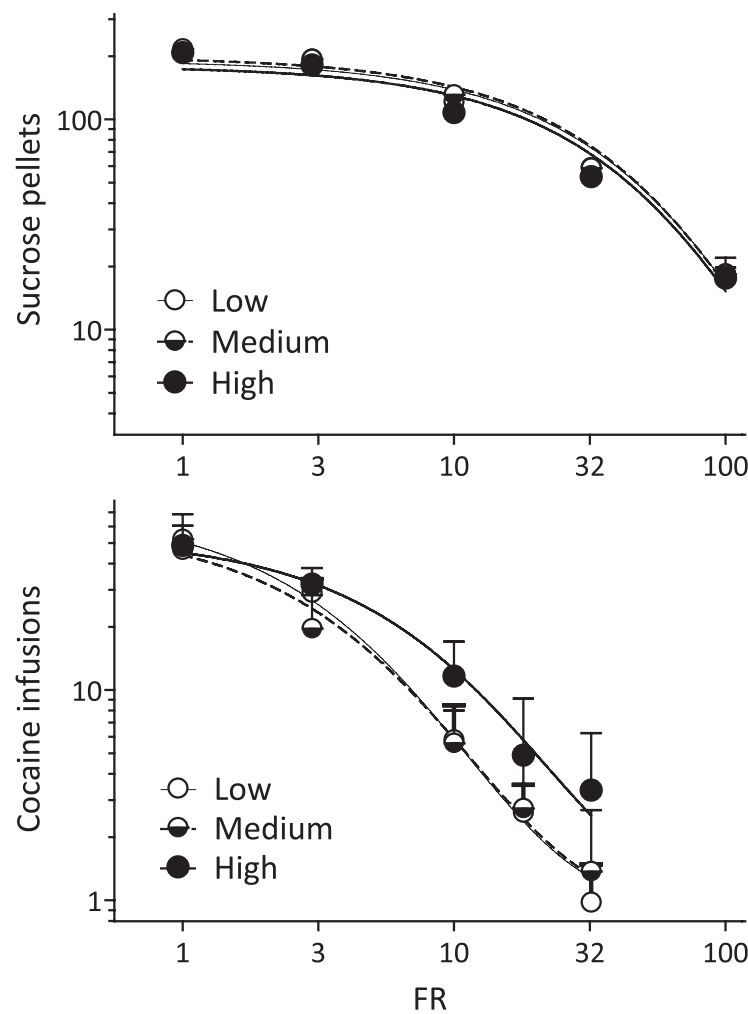

Figure 3 Demand curves. Top panel: Demand for sucrose pellets as a function of discounting group. Data are plotted as consumption of sucrose pellets as a function of fixed-ratio value. Bottom panel: Demand for cocaine injections as a function of discounting group

approximated by Equation 2 (median $r^{2}=0.962$, interquartile range $=0.060$ ). The mean index of curvature for cocaine self-administration sessions was -0.11 , indicating a slight deceleration of responding within the 


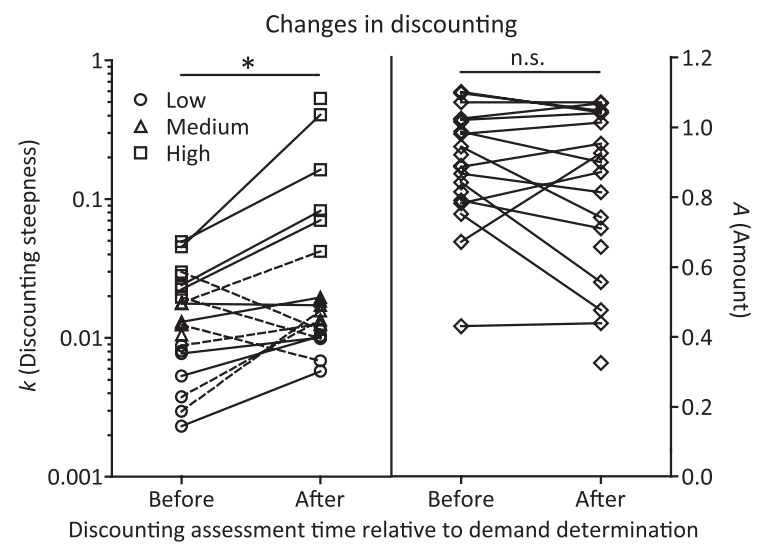

Figure 4 Changes in discounting parameters from the initial discounting assessment to the discounting reassessment. The shapes of the symbols in the left panel indicate the group subject was placed into for the initial assessment before demand evaluation and the reassessment after demand evaluation. Solid lines connect points for a single subject when that subject stayed in the same group, while dotted lines connect points for subjects that switched groups from one assessment to the next. The results of statistical comparisons between time points are also shown $(* P<0.05 ;$ n.s. $=$ not significant $)$

sessions. This value did not differ between sucrose and cocaine sessions $\left(t_{19}=0.32, P=0.751\right)$.

\section{Phase 4: discounting reassessment}

Data from the discounting reassessment were analyzed to determine if the discounting parameters changed following sucrose and cocaine self-administration and whether $k$ values obtained from the discounting reassessment were also associated with cocaine demand. Twenty-one rats remained at the end of the cocaine demand determination. Of these, 18 met the statistical criteria for inclusion in a second set of discounting groups. Two of the rats that failed to meet criteria also did not meet criteria in the initial assessment. The other rat met criteria in the original assessment but failed to meet criteria in the reassessment. The other two of the four rats that failed to meet criteria in the original assessment did meet criteria in the reassessment.

The discounting reassessment curves, in general, appeared to be steeper than those in the initial discounting assessment (see Supporting Information Fig. S2, bottom panel). This was confirmed by a paired $t$-test on the $\log k$ values from the individual curves in the initial assessment and reassessment (Fig. 4; $t_{15}=2.8, \quad P=$ 0.013). The $A$ values from Equation 1 did not differ between discounting assessments (Fig. 4; $t_{15}=1.1$, $P=0.306)$. Any changes noted between the initial discounting assessment and reassessment did not depend on initial discounting group for $\log k$ values $\left(F_{2,13}=0.78\right.$, $P=0.480)$ or $A$ values $\left(F_{2,13}=0.43, P=0.662\right)$. In those rats that completed and met significance criteria for inclusion in both discounting assessments, the respective $k$ and $A$ values from Equation 1 were significantly correlated across assessments ( $\log k$ correlation $r=0.698$, $n=16, \quad P=0.003 ; A$ correlation $r=0.770, n=16$, $P<0.001)$.

If the 18 rats that met criteria in the reassessment were again split into three groups of six rats each using $k$ from Equation 1 fit to the individual choice data (median $r^{2}=0.879$, interquartile range $=0.133$ ), nine of the rats that met criteria of both assessments stayed in the same discounting group, while seven switched groups. Since assignment to groups was based on rank order $k$ values, a switch among groups was not necessarily indicative of an increase or decrease in $k$ value for that subject, but rather a change in rank compared with the other subjects. Analyzed by group in these 18 subjects, there was a main effect of delay $\left(F_{4,60}=24, P<0.001\right)$ and discounting group $\left(F_{2,60}=48, P<0.001\right)$ on choices of three pellets (Supporting Information Fig. S2, top panel). These differences also tended to be larger at the higher delays, leading to a significant group by delay interaction $\left(F_{8,60}=3.8\right.$, $P=0.001)$. Note that in the discounting reassessment, the choices of the groups were not equal in the 0-second delay component.

If performance in the discounting reassessment was used as a basis for group selection, the effect of discounting group on demand for cocaine remained, and there was still no difference in sucrose demand (Table 2; Supporting Information Fig. S3). This indicates that it did not matter if discounting was assessed prior to or following demand; the same pattern of results remained (association with cocaine demand, but not with sucrose demand).

\section{DISCUSSION}

Subjects appeared to learn the contingencies of the operative schedule of reinforcement during each phase of the current experiment, with data largely following the expected patterns. Individual differences in delay discounting did not predict level or elasticity of demand for sucrose, but did predict elasticity of demand for self-administered cocaine injections. This relationship occurred whether delay discounting was measured prior to or after demand assessments.

Sizeable individual differences in sensitivity to delay produced discounting groups that significantly differed from one another in their choices of immediate and delayed rewards (Fig. 2). Individual $\log k$ and $A$ values correlated between the initial discounting assessment and reassessment (Fig. 4), But overall, discounting was steeper in the second assessment. It should be noted that Equation 1, used here to differentiate subjects based on 
Table 2 A comparison of the demand parameters for each group of rats split on the basis of the individual $k$ values obtained from the discounting reassessment. Normalized and standard demand analyses are presented with both sucrose and cocaine reinforcers, as well as the results of $F$ tests comparing these parameters across groups. Statistically significant results appear in bold.

\begin{tabular}{|c|c|c|c|c|c|c|c|}
\hline & \multirow[b]{2}{*}{ Parameter } & \multicolumn{3}{|c|}{ Discounting reassessment group } & \multirow[b]{2}{*}{$\mathrm{F}$} & \multirow{2}{*}{$\begin{array}{l}\text { Degree of } \\
\text { freedom }\end{array}$} & \multirow[b]{2}{*}{$\mathrm{P}$} \\
\hline & & Low & Medium & High & & & \\
\hline \multirow[t]{3}{*}{ Sucrose: not normalized } & $L$ & 187 & 194 & 202 & 0.312 & 2,84 & 0.733 \\
\hline & $a$ & 0.00615 & 0.00613 & 0.00626 & 0.0271 & 2,84 & 0.973 \\
\hline & $P_{\max }$ & 47.2 & 47.3 & 46.3 & & & \\
\hline \multirow[t]{2}{*}{ Sucrose: normalized } & $a$ & 0.000175 & 0.000217 & 0.000245 & 0.115 & 2,87 & 0.892 \\
\hline & $P_{\max }$ & 75.2 & 77.3 & 78.1 & & & \\
\hline \multirow[t]{3}{*}{ Cocaine: not normalized } & $L$ & 65.6 & 63.5 & 60.1 & 0.0144 & 2,69 & 0.986 \\
\hline & $a$ & 0.0953 & 0.0545 & 0.0440 & 3.19 & 2,69 & 0.047 \\
\hline & $P_{\max }$ & 3.04 & 5.32 & 6.60 & & & \\
\hline \multirow[t]{2}{*}{ Cocaine: normalized } & $a$ & 0.121 & 0.0720 & 0.0608 & 5.82 & 2,72 & 0.005 \\
\hline & $P_{\max }$ & 2.40 & 4.03 & 4.77 & & & \\
\hline
\end{tabular}

$a=$ Demand elasticity parameter from Equation 2; $L=$ Parameter representing level of responding from Equation $2 ; P_{\max }=$ Price supporting the maximum level of responding.

sensitivity to delay, is not typically used to analyze data obtained from the Evenden \& Ryan (1996) procedure. For $k$ to be a true representation of discounting rate as proposed by Mazur (1987), a series of indifferent points assessed with distinct amount and delay comparisons should first be obtained. Only one amount comparison was included in the current experiment (i.e. one versus three sucrose pellets), and therefore only one indifference point could be obtained from any subject's choice data. Curve-fitting with a single datum point is of little use. Rather, this hyperbolic function was used because it happened to describe the choice data in the current experiment well, and provided a simple one-parameter assessment of sensitivity to delay $(k)$ and amount $(A)$. To determine if the findings of the current experiment were a by-product of the specific equation chosen to summarize the obtained data, the slope and $y$-intercept of linear regression analyses drawn through choice data were also obtained and used as measurements of sensitivity to delay (slope) and amount ( $y$-intercept). While this method of grouping subjects did not result in an identical composition of the high, medium and low groups, statistical conclusions of data described in this manner were not appreciably different and did not lead to different logical conclusions (i.e. the linear regression high group had significantly less elastic demand for cocaine than the linear regression low group, with no difference in sucrose demand).

Individual differences in discounting were associated with elasticity of demand for cocaine (Fig. 3), suggesting that impulsive choice differentiates assessments of cocaine value. An analogous relationship was not found between impulsive choice and demand for sucrose (Fig. 3), indicating that differences in cocaine demand were not simply due to differences in propensity to respond for reinforcers of any type or overall level of responding. Instead, a specific relationship seems to exist between cocaine demand and impulsive choice. Previous research has suggested a relationship between impulsive choice and acquisition of cocaine self-administration in female rats (Perry et al. 2005). The current research extends this finding by relating elasticity of cocaine demand in male rats to impulsive choice, with a sucrose demand control condition. It is notable that level of cocaine demand ( $L$ from Equation 2 ), which approximates responding on an FR 1, did not differ between delay discounting groups. Only elasticity of demand differed, which agrees with the finding that delay discounting is associated with nicotine self-administration at high FR values only (Diergaarde et al. 2008). Analogous to the current experiment, behavioral economic demand analyses have been conducted in human nicotine-dependent adults (MacKillop \& Tidey 2011) and alcohol users (MacKillop et al. 2010). Among nicotine-independent adults (MacKillop \& Tidey 2011), demand elasticity was correlated with delay discounting of a small monetary reward, but the same relationship was not found among alcohol users (MacKillop et al. 2010). A similar study has not been conducted in cocaine users.

Both delay discounting and demand analysis purport to assess different contributors to the value of a reinforcer. Delay discounting assesses how a delay to the delivery of a reinforcer influences value as measured by choice behavior, while demand analysis assesses the consumption of a reinforcer across a range of prices or response requirements and is thought to measure the intrinsic value inherent to a specific reinforcer, sometimes called 'essential value’ (Hursh \& Silberberg 2008). Given 
that both procedures measure value, it is notable that discounting of delayed sucrose pellets is not related to demand for sucrose pellets but is related to demand for the distinct reinforcer cocaine. This pattern of results suggests that delay discounting is measuring a separate and distinct contributor to value than is demand analysis, and the results of the current experiment are not simply a by-product of measuring value in two different ways. Instead, the relationship between discounting of delayed sucrose pellets and elasticity of demand for cocaine seems to be more similar to the analogous pattern of results seen with human cocaine abusers who exhibit greater discounting of delayed money, a situation where the reinforcers for each measure also differ (Coffey et al. 2003; Kirby \& Petry 2004).

Due to the inherent limitations of human-subjects research, the causal direction, if any, of the correlation between cocaine abuse and delay discounting in humans has not been conclusively determined. Four explanations for this observed correlation are plausible: inborn variability in impulsive choice predisposes an individual to an increased likelihood of abusing cocaine, cocaine exposure and the life experiences associated with procuring and consuming cocaine increase impulsive choice, both characteristics cause the other in a positive-feedback loop, or both impulsive choice and cocaine abuse vulnerability are caused by an unknown third variable and do not otherwise interact. Limited evidence exists demonstrating that impulsivity, as measured by personality questionnaires and behavioral assessments, predicts development of drug abuse in human subjects (for review, see de Wit 2009). Perhaps most relevant to impulsive choice is a single study finding that individual differences in delay discounting assessed at grade 10 predicts initiation of smoking within the following 4 years (Audrain-McGovern et al. 2009). The results of the current experiment, to the extent that the models employed simulate the human condition, suggest that such a positive relationship does occur between natural variability in impulsive choice and propensity to selfadminister cocaine.

Whether the act of abusing a substance increases discounting has not been studied experimentally in humans for obvious reasons. However, acute drug effects have been shown to affect impulsive choice (for review, see de Wit 2009) and recent opioid intake influences level of discounting in opioid abusers (Giordano et al. 2002). However, ex-smokers and never-smokers discount delayed rewards similarly and less than current smokers, suggesting that if smoking alters impulsive choice, it does so temporarily (Bickel, Odum \& Madden 1999). Delay discounting assessments conducted before and after the initiation of smoking also did not find evidence that smoking altered discounting of delayed rewards
(Audrain-McGovern et al. 2009), despite discounting in smokers being higher (Bickel et al. 1999; Mitchell 1999; Baker, Johnson \& Bickel 2003; Heyman \& Gibb 2006; Reynolds 2006; Johnson, Bickel \& Baker 2007; Reynolds et al. 2007; Jones et al. 2009). These results suggest that impulsive choice may predict smoking, but not vice versa. The current study was not designed optimally to determine if cocaine self-administration influences discounting, but an increase in impulsive choice was measured from the initial discounting assessment to the discounting redetermination assessed after a period of cocaine self-administration. Increased impulsive choice with age is not typical, as impulsive choice typically negatively correlated with age in people (Green, Fry \& Myerson 1994) and in rats (Simon et al. 2010). An appropriate control group that experienced all the behavioral and surgical components of the current experiment without cocaine self-administration was not included, however. This trend does agree with previous research showing noncontingent injections (Logue et al. 1992; Paine et al. 2003; Simon et al. 2007) and self-administered injections (Mendez et al. 2010) of cocaine lead to a subsequent increase in impulsive choice.

Procedural details in the current experiment that potentially affected results included the use of an ascending delay sequence in the delay discounting task and an ascending series of FR values in the two demand determinations. However, it is unlikely that an ascending order of delays and FR values were influential, because ascending delays in the delay discounting task result in similar discounting rates as descending delays (Slezak \& Anderson 2009), and an ascending series of FR values result in similar demand curves as a random FR sequence (Raslear et al. 1988). An additional potential problem with measuring the 'essential value' of a reinforcer involves the somewhat arbitrary limit set on session length of the demand sessions (30 minutes) and the potential that that marginal value of each additional reinforcer changed within demand sessions in a way that differed between cocaine and sucrose sessions, biasing results. To assess whether the marginal value of each successive reinforcer changed within a session, index of curvature values were collected that quantified the positive or negative acceleration of response rates within sessions. Mean index of curvature values for sucrose sessions $(-0.10)$ and cocaine sessions $(-0.11)$ did not differ, suggesting that the marginal value of each successive sucrose pellet and cocaine injection may have decreased somewhat within a session, but this decrease was very similar across reinforcers. Since the pattern of response rates within a session was similar across reinforcers, it is unlikely that changes in marginal value biased the measures of essential value that were obtained in the demand analyses. 
In conclusion, individual differences in impulsive choice are associated with elasticity of cocaine demand, a measure of reinforcer value. This relationship holds if impulsive choice is measured before or after cocaine demand is determined and sucrose demand is not differentiated by individual differences in impulsive choice. Impulsive choice was increased following cocaine selfadministration, but the cause of this increase cannot be conclusively identified by the current experiment.

\section{Acknowledgements}

The authors would like to thank Davina Barron, Aaron Chadderdon, Alexa Cohen, Bruce Kaczmarek, Kevin Kammel, Collette Rothe, Sarah Snider and Nhu Truong for technical assistance, and Gail Winger, Jeremiah Bertz and Nhu Truong for advice and help with manuscript preparation. This research was supported by NIH/National Institute on Drug Abuse (NIDA) grants R01 DA015449, R01 DA020669 and T32 DA007267. The content is solely the responsibility of the authors and does not necessarily represent the official views of NIDA or the NIH.

\section{Author Contributions}

MNK conceived the study, carried it out, analyzed the data and drafted the manuscript. JHW provided critical revision of the manuscript for important intellectual content. Both authors critically reviewed content and approved final version for publication.

\section{REFERENCES}

Ainslie G (1975) Specious reward: a behavioral theory of impulsiveness and impulse control. Psychol Bull 82:463496.

Audrain-McGovern J, Rodriguez D, Epstein LH, Cuevas J, Rodgers K, Wileyto EP (2009) Does delay discounting play an etiological role in smoking or is it a consequence of smoking? Drug Alcohol Depend 103:99-106.

Baker F, Johnson MW, Bickel WK (2003) Delay discounting in current and never-before cigarette smokers: similarities and differences across commodity, sign, and magnitude. J Abnorm Psychol 112:382-392.

Bergman J, Paronis CA (2006) Measuring the reinforcing strength of abused drugs. Mol Interv 6:273-283.

Bickel WK, Green L, Vuchinich RE (1995) Behavioral economics. J Exp Anal Behav 64:257-262.

Bickel WK, Odum AL, Madden GJ (1999) Impulsivity and cigarette smoking: delay discounting in current, never, and ex-smokers. Psychopharmacology 146:447-454.

Coffey SF, Gudleski GD, Saladin ME, Brady KT (2003) Impulsivity and rapid discounting of delayed hypothetical rewards in cocaine-dependent individuals. Exp Clin Psychopharmacol $11: 18-25$.

Dalley JW, Mar AC, Economidou D, Robbins TW (2008) Neurobehavioral mechanisms of impulsivity: fronto-striatal systems and functional neurochemistry. Pharmacol Biochem Behav 90:250-260.
Diergaarde L, Pattij T, Poortvliet I, Hogenboom F, de Vries W, Schoffelmeer ANM, De Vries TJ (2008) Impulsive choice and impulsive action predict vulnerability to distinct stages of nicotine seeking in rats. Biol Psychiat 63:301308.

Evenden J (1999) Impulsivity: a discussion of clinical and experimental findings. J Psychopharmacol 13:180-192.

Evenden JL, Ryan CN (1996) The pharmacology of impulsive behaviour in rats: the effects of drugs on response choice with varying delays of reinforcement. Psychopharmacology 128:161-170.

Fry W, Kelleher RT, Cook L (1960) A mathematical index of performance on fixed-interval schedules of reinforcement. J Exp Anal Behav 3:193-199.

Giordano LA, Bickel WK, Goewenstein G, Jacobs EA, Marsch L, Badger GJ (2002) Mild opioid deprivation increases the degree that opioid-dependent outpatients discount delayed heroin and money. Psychopharmacology 163:174-182.

Green L, Fry AF, Myerson J (1994) Discounting of delayed rewards: a life-span comparison. Psychol Sci 5:33-36.

Heyman GM, Gibb SP (2006) Delay discounting in cigarette chippers. Behav Pharmacol 17:669-679.

Hursh SR (1991) Behavioral economics of drug selfadministration and drug abuse policy. J Exp Anal Behav 56:377-393.

Hursh SR, Silberberg A (2008) Economic demand and essential value. Psychol Rev 115:186-198.

Hursh SR, Winger G (1995) Normalized demand for drugs and other reinforcers. J Exp Anal Behav 64:373-384.

Johnson MW, Bickel WK (2008) An algorithm for identifying nonsystematic delay-discounting data. Exp Clin Psychopharmacol 16:264-274.

Johnson MW, Bickel WK, Baker F (2007) Moderate drug use and delay discounting: a comparison of heavy, light, and never smokers. Exp Clin Psychopharmacol 15:187-194.

Jones BA, Landes RD, Yi R, Bickel WK (2009) Temporal horizon: modulation by smoking status and gender. Drug Alcohol Depend 104:S87-S93.

Kirby KN, Petry NM (2004) Heroin and cocaine abusers have higher discount rates for delayed rewards than alcoholics or non-drug-using controls. Addiction 99:461-471.

Logue AW, Tobin H, Chelonis JJ, Wang RY, Geary N, Schachter S (1992) Cocaine decreases self-control in rats: a preliminary report. Psychopharmacology 109:245-247.

MacKillop J, Tidey JW (2011) Cigarette demand and delayed reward discounting in nicotine-dependent individuals with schizophrenia and controls: an initial study. Psychopharmacology 216:91-99.

MacKillop J, Miranda R, Monti PR, Ray LA, Murphy JG, Rohsenow DJ, McGeary JE, Swift RM, Tidey JW, Gwaltney CJ (2010) Alcohol demand, delayed reward discounting, and craving in relation to drinking and alcohol use disorders. J Abnorm Psychol 119:106-114.

Mazur JE (1987) An adjusting procedure for studying delayed reinforcement. In: Commons ML, Mazur JE, Nevin JA, Rachlin H, eds. Quantitative Analysis of Behavior (Vol. 5) The Effect of Delay and of Intervening Events on Reinforcement Value, pp. 55-73. Hillsdale, NJ: Erlbaum.

Mendez IA, Simon NW, Hart N, Mitchell MR, Nation JR, Wellman PJ, Setlow B (2010) Self-administered cocaine causes long-lasting increases in impulsive choice in a delay discounting task. Behav Neurosci 124:470-477.

Mitchell SH (1999) Measures of impulsivity in cigarette smokers and non-smokers. Psychopharmacology 146:455-464. 
Motulsky HJ, Christopoulos A (2003) Fitting Models to Biological Data Using Linear and Nonlinear Regression: A Practical Guide to Curve Fitting. San Diego, CA: GraphPad Software, Inc.

Paine TA, Dringenberg HC, Olmstead MC (2003) Effects of chronic cocaine on impulsivity: relation to cortical serotonin mechanisms. Behav Brain Res 147:135-147.

Perry JL, Carroll ME (2008) The role of impulsive behavior in drug abuse. Psychopharmacology 200:1-26.

Perry JL, Larson EB, German JP, Madden GJ, Carroll ME (2005) Impulsivity (delay discounting) as a predictor of acquisition of IV cocaine self-administration in female rats. Psychopharmacology 178:193-201.

Raslear TG, Bauman RA, Hursh SR, Shurtleff D, Simmons L (1988) Rapid demand curves for behavioral economics. Anim Learn Behav 1:223-227.

Reynolds B (2006) The experiential discounting task is sensitive to cigarette-smoking status and correlates with a measure of delay discounting. Behav Pharmacol 17:133-142.

Reynolds B, Patak M, Shroff P, Penfold RB, Melanko S, Duhig AM (2007) Laboratory and self-report assessments of impulsive behavior in adolescent daily smokers and nonsmokers. Exp Clin Psychopharmacol 15:264-271.

Richards JB, Mitchell SH, de Wit H, Seiden LS (1997) Determination of discount functions in rats with an adjusting-amount procedure. J Exp Anal Behav 67:353-366.

Simon NW, Lasarge CL, Montgomery KS, Williams MT, Mendez IA, Setlow B, Bizon JL (2010) Good things come to those who wait: attenuated discounting of delayed rewards in aged Fischer 344 rats. Neurobiol Aging 31:853-862.

Simon NW, Mendez IA, Setlow B (2007) Cocaine exposure causes long-term increases in impulsive choice. Behav Neurosci 121:543-549.
Slezak JM, Anderson KG (2009) Effects of variable training, signaled and unsignaled delays, and $d$-amphetamine on delay-discounting functions. Behav Pharmacol 20:424436.

Winger G, Galuska CM, Hursh SR, Woods JH (2006) Relative reinforcing effects of cocaine, remifentanil, and their combination in rhesus monkeys. J Pharmacol Exp Ther 318:223229.

Winstanley CA, Eagle DM, Robbins TW (2006) Behavioral models of impulsivity in relation to ADHD: translation between clinical and preclinical studies. Clin Psychol Rev 26:379-395.

de Wit H (2009) Impulsivity as a determinant and consequence of drug use: a review of underlying processes. Addict Biol 14:22-31.

\section{SUPPORTING INFORMATION}

Additional Supporting Information may be found in the online version of this article:

Figure S1 Normalized demand. Top panel: Normalized demand for sucrose pellets as a function of discounting group.

Figure S2 Choice data from the delay discounting reassessment.

Figure S3 Normalized demand plotted as a function of discounting groups determined by the discounting reassessment. 